\title{
Clinical role of pleural effusion MMP-3 levels in malignant pleural mesothelioma
}

\author{
AKI MURAKAMI $^{*}{ }^{*}$, CHIHARU TABATA $^{1 *}$, RIE TABATA $^{2}$, HISAYA OKUWA $^{1}$ and TAKASHI NAKANO ${ }^{1}$ \\ ${ }^{1}$ Division of Respiratory Medicine, Department of Internal Medicine, Hyogo College of Medicine; \\ ${ }^{2}$ Department of Internal Medicine, Hyogo Prefectural Tsukaguchi Hospital, Hyogo 663-8501, Japan
}

Received October 3, 2011; Accepted November 25, 2011

DOI: 10.3892/ol.2011.536

\begin{abstract}
Malignant pleural mesothelioma (MPM) is an aggressive malignant tumor of mesothelial origin associated with asbestos exposure. MPM exhibits a limited response to conventional chemotherapy and radiotherapy. This, early diagnosis of MPM is essential. Malignant tumor progression requires the destruction of the basement membrane, which is constructed from extracellular matrix (ECM) materials. Various types of human tumor cells are reported to produce ECM-degrading proteases that are important in tumor progression. Among this group of proteolytic enzymes, matrix metalloproteinases (MMPs) are thought to be important due to their wide degrading function. We investigated the pleural effusion MMP-3 levels of patients with MPM and compared them with those of a population with non-malignant pleuritis or lung cancer involving malignant pleural effusion. The pleural effusion MMP-3 concentrations of 52 MPM patients and 67 non-MPM patients were measured. The results showed that the MPM patients had significantly higher pleural effusion MMP-3 levels than the population with non-malignant pleuritis. The overall survival of the MPM patients with lower pleural effusion MMP-3 levels was longer than that of patients with higher pleural effusion MMP-3 levels. Our data therefore suggest a clinical role of pleural effusion MMP-3 levels in malignant pleural mesothelioma.
\end{abstract}

Correspondence to: Dr Chiharu Tabata, Division of Respiratory Medicine, Department of Internal Medicine, Hyogo College of Medicine, 1-1 Mukogawa-cho, Nishinomiya, Hyogo 663-8501, Japan E-mail: ctabata@hyo-med.ac.jp

*Contributed equally

Abbreviations: AUC, area under the ROC curve; BM, basement membrane; CI, confidence interval; ECM, extracellular matrix; ELISA, enzyme-linked immunosorbent assay; IL, interleukin; MMP, matrix metalloproteinase; MPM, malignant pleural mesothelioma; PDGF, platelet-derived growth factor; ROC, receiver operating characteristic; TGF, transforming growth factor

Key words: asbestos-related lung diseases, malignant mesothelioma, tumor marker, diagnosis, prognosis

\section{Introduction}

Malignant pleural mesothelioma (MPM) is an aggressive malignant tumor of mesothelial origin associated with asbestos exposure (1-3). The lifetime risk of MPM is associated with a history of occupational and/or environmental asbestos exposure (4). Due to the long latency period (typically over 30 years) between the first asbestos exposure and the onset of the disease, MPM remains a universally fatal disease of increasing incidence worldwide $(1,2,5)$, although asbestos usage has recently decreased in Western countries and Japan.

Malignant tumor progression requires the destruction of the basement membrane (BM), which is constructed from extracellular matrix (ECM) materials. Various human tumor cells are reported to produce ECM-degrading proteases that are important in tumor progression (6). Among this group of proteolytic enzymes, matrix metalloproteinases (MMPs) are thought to be important due to their wide degrading function. MMPs are zinc-dependent endopeptidases, whose activities are targeted to all components of the ECM (7).

MMP-3 is known to be involved in tumor cell invasion and metastasis (8). The increased expression of MMP-3 has been reported in several malignant tumors, including esophageal cancer (9), breast cancer (10) and glioma (11). Moreover, a correlation between a higher MMP-3 expression and disease progression has been reported in patients with gastric cancer (12), hepatocellular carcinoma (13) and bladder cancer (14). However, the clinical importance of MMP-3 in MPM patients has not been fully investigated, although MMP-3 expression has been reported in certain MPM cells $(15,16)$. In this study, we evaluated the clinical role of the pleural effusion MMP-3 concentration as a biomarker in MPM.

\section{Materials and methods}

Patients and pleural effusion samples. The MMP-3 levels in pleural effusion samples collected from 119 individuals presenting at the Department of Respiratory Medicine of Hyogo College of Medicine between 2005 and 2009 were examined. The pleural effusions were obtained by thoracocentesis. All cases were diagnosed by pathologists, and it was confirmed that their clinical course matched their diagnosis. Fifty-two individuals had MPM involving a documented asbestos exposure history. These cases were diagnosed by patholo- 
gists skilled in the diagnosis of MPM using histopathological samples. The patients were classified using the staging system of the International Mesothelioma Interest Group (IMIG) (17). Patients with MPM were treated according to our therapeutic guidelines: combination chemotherapy including the multi-target anti-folate pemetrexed was performed for patients with performance status (PS) $0-1$ who were aged $<70$, and the best supportive care was selected for the remaining patients. Surgical treatment was not performed in any patient in the present study. Thirty-three individuals, including 8 cases with benign asbestos pleurisy, had non-malignant pleural effusion. Thirty-four individuals had lung cancer involving malignant pleural effusion without asbestos exposure. We verified the asbestos exposure by interview. The study was approved by our ethics committee in accordance with the 1975 Declaration of Helsinki. Informed consent was obtained from all patients. Fresh pleural effusion samples were collected prior to treatment and centrifuged for $10 \mathrm{~min}$ at $2000 \mathrm{x} \mathrm{g}$ at $4^{\circ} \mathrm{C}$, and the resultant supernatants were immediately frozen in liquid nitrogen and stored at $-80^{\circ} \mathrm{C}$ until use.

Measurement of MMP-3. The MMP-3 concentrations of the pleural effusions were measured using an enzyme-linked immunosorbent assay (ELISA) kit (R\&D Systems, Oxford, $\mathrm{UK}$ ) according to the manufacturer's instructions.

Statistical analysis. The non-parametric Mann-Whitney U test was used to compare three groups of samples. In all tests, $\mathrm{p}<0.05$ was considered to indicate a statistically significant result. To estimate the significance of the pleural effusion MMP-3 values, receiver operating characteristic (ROC) curves, the area under the ROC curve (AUC) and their 95\% confidence intervals $(95 \% \mathrm{CI})$ were calculated using standard techniques. To examine the cut-off values for pleural effusion MMP-3 levels, we calculated the total sensitivity and specificity for each cut-off value and then selected the cut-off values that maximized each factor. Estimates of the probability of survival were calculated by the Kaplan-Meier method and compared using the log-rank test to evaluate the prognostic significance of MMP-3 with regard to the survival of patients with MPM.

\section{Results}

MMP-3 pleural effusion levels in MPM and non-MPM patients. We recruited a total of 119 subjects presenting with pleural effusion. Of the 119 patients, 52 had confirmed MPM, 33 had non-malignant pleural effusion, and 34 had lung cancer involving malignant pleural effusion. Their characteristics are shown in Table I. Of the 52 patients with MPM, 40 were of epithelioid histology, 10 sarcomatoid and 2 biphasic.

The ROC curves for pleural effusion MMP-3 levels (Fig. 1A) reveal that the patients with MPM had an AUC of 0.651 in comparison with those with non-malignant pleural effusion (95\% CI 0.555-0.747). At the optimal cut-off value of $50 \mathrm{ng} / \mathrm{ml}$, the diagnostic sensitivity was $30.8 \%$ and the specificity was 97.0\%. The mean pleural effusion MMP-3 concentration of the patients with MPM was significantly higher $(49.1 \pm 59.1 \mathrm{ng} / \mathrm{ml})$ than that of the patients with non-malignant pleural effusion $(19.8 \pm 13.8 \mathrm{ng} / \mathrm{ml}$; $\mathrm{p}=0.028$; Fig. 1B). Although the mean
Table I. Characteristics of the MPM and non-MPM patients.

\begin{tabular}{|c|c|c|}
\hline Patient group & Cases $(\%)$ & Total \\
\hline MPM & & 52 \\
\hline Age, years (mean $\pm \mathrm{SD}$ ) & $69.1 \pm 10.3$ & \\
\hline \multicolumn{3}{|l|}{ Gender } \\
\hline Male & $39(75.0)$ & \\
\hline Female & $13(25.0)$ & \\
\hline \multicolumn{3}{|l|}{ Histology } \\
\hline Epithelioid & $40(76.9)$ & \\
\hline Sarcomatoid & $10(19.2)$ & \\
\hline Biphasic & $2(3.9)$ & \\
\hline \multicolumn{3}{|l|}{ Stage } \\
\hline I & $8(15.4)$ & \\
\hline II & $5(9.6)$ & \\
\hline III & $8(15.4)$ & \\
\hline IV & $31(59.6)$ & \\
\hline Non-malignant & & 33 \\
\hline Age, years $($ mean $\pm S D)$ & $70.6 \pm 11.1$ & \\
\hline \multicolumn{3}{|l|}{ Gender } \\
\hline Male & $28(84.8)$ & \\
\hline Female & $5(15.2)$ & \\
\hline \multicolumn{3}{|l|}{ Histology } \\
\hline Benign asbestos pleurisy & $8(24.1)$ & \\
\hline Tuberculous ( $\mathrm{Tb}$ ) pleurisy & $9(27.3)$ & \\
\hline Infectious (non-Tb) pleurisy & $9(27.3)$ & \\
\hline Empysema & $2(6.1)$ & \\
\hline Heart failure & $2(6.1)$ & \\
\hline Hepatic failure & $1(3.0)$ & \\
\hline Renal failure & $2(6.1)$ & \\
\hline Lung cancer & & 34 \\
\hline Age, years $($ mean $\pm S D)$ & $67.6 \pm 11.1$ & \\
\hline \multicolumn{3}{|l|}{ Gender } \\
\hline Male & $23(67.6)$ & \\
\hline Female & $11(32.4)$ & \\
\hline \multicolumn{3}{|l|}{ Histology } \\
\hline Adenocarcinoma & $31(91.2)$ & \\
\hline Squamous cell carcinoma & $2(5.9)$ & \\
\hline Small cell carcinoma & $1(2.9)$ & \\
\hline
\end{tabular}

MPM, malignant pleural mesothelioma.

pleural effusion MMP-3 concentrations of the patients with MPM was higher than that of the patients with lung cancer involving malignant pleural effusion $(33.7 \pm 22.4 \mathrm{ng} / \mathrm{ml})$, no statistically significant difference was found between them ( $p=0.909$; Fig. 1B). No statistically significant differences were observed between the pleural effusion MMP-3 levels of the MPM histological groups (epithelioid, $54.2 \pm 62.9 \mathrm{ng} / \mathrm{ml}$; non-epithelioid, $32.1 \pm 45.5 \mathrm{ng} / \mathrm{ml}$ ) or the different disease stages (stage I, $33.1 \pm 30.2 \mathrm{ng} / \mathrm{ml}$; stage II, $34.0 \pm 32.2 \mathrm{ng} / \mathrm{ml}$; stage III, $84.6 \pm 120.3 \mathrm{ng} / \mathrm{ml}$; and stage IV, $49.9 \pm 58.8 \mathrm{ng} / \mathrm{ml}$ ) and there were no significant differences between the pleural 
A
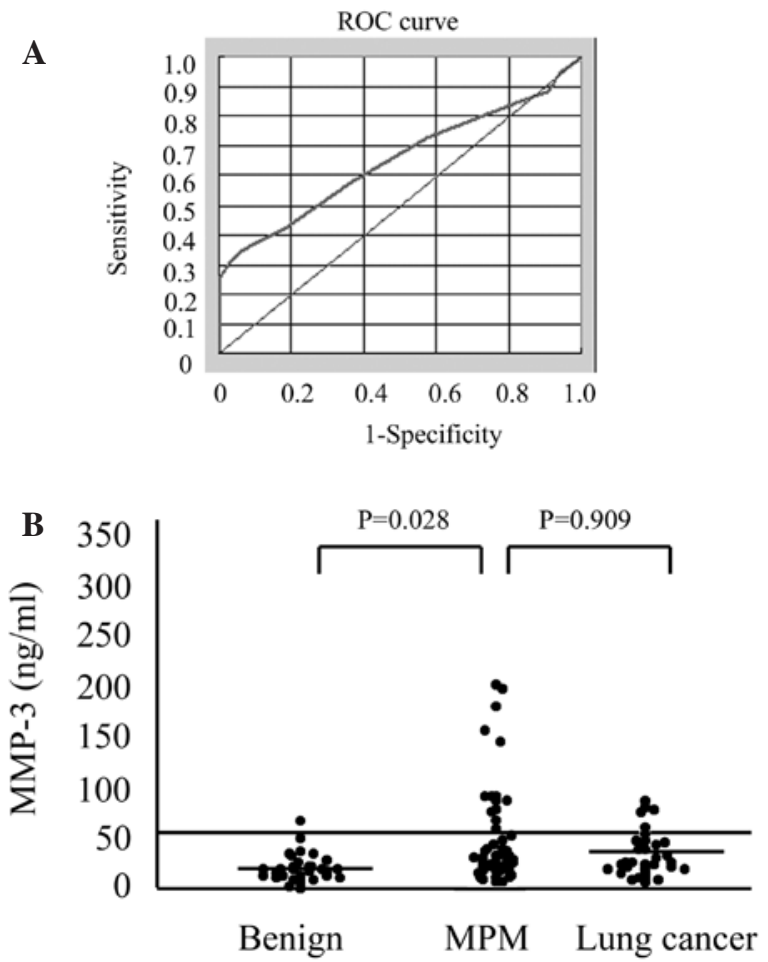

Figure 1. Pleural effusion MMP-3 levels in patients with MPM and patients with non-MPM. (A) An analysis that included 52 MPM and 33 non-malignant pleural effusion patients revealed an area under the curve (AUC) of 0.651 (95\% CI 0.555-0.747). At the optimal cut-off value of $50 \mathrm{ng} / \mathrm{ml}$, the diagnostic sensitivity was $30.8 \%$ and the specificity was $97.0 \%$. (B) The pleural effusion MMP-3 levels of the patients with MPM versus those of the patients with non-malignant pleural effusion or lung cancer involving malignant pleural effusion were measured as described in Materials and methods. The nonparametric Mann-Whitney $U$ test was used, and $p<0.05$ was considered to indicate a statistically significant result. The horizontal bars are the mean for each group. The cut-off value is shown as a horizontal line. ROC, receiver operating characteristic; MPM, malignant pleural mesothelioma; MMP-3, matrix metalloproteinase-3; CI, confidence interval.

effusion MMP-3 levels of the subjects with benign asbestos pleurisy and those with benign pleurisy without a history of asbestos exposure $(14.4 \pm 9.0 \mathrm{ng} / \mathrm{ml}$ and $20.3 \pm 14.2 \mathrm{ng} / \mathrm{ml}$, respectively).
Correlation between pleural effusion MMP-3 levels and overall survival. Among the 52 MPM patients, we were able to follow 47 patients closely for up to 1,700 days. Twenty patients had died by the end of the follow-up. Five patients were lost due to lack of information following transfer to other hospitals. These 5 subjects were homogenously distributed in the two groups compared ( 3 subjects were $>50 \mathrm{ng} / \mathrm{ml}$ and 2 subjects were $\leq 50 \mathrm{ng} / \mathrm{ml})$.

To study the correlation between pleural effusion MMP-3 levels and patient clinical courses, we separated the patients according to their pleural effusion MMP-3 levels at the time of the first measurement. The first group included patients with pleural effusion MMP-3 levels lower than $50 \mathrm{ng} / \mathrm{ml}$, the cut-off value that we selected. In this group of 34 patients, the mean MMP-3 value was $18.2 \mathrm{ng} / \mathrm{ml}$ (interquartile range, 10.5-26.2). The other group included the remaining 13 patients, who had pleural effusion MMP-3 levels higher than $50 \mathrm{ng} / \mathrm{ml}$ and whose mean MMP-3 pleural effusion concentration was $109.5 \mathrm{ng} / \mathrm{ml}$ (interquartile range, 71.8-139.1). The difference in overall survival between the groups with lower and higher pleural effusion MMP-3 concentrations than the cut-off point of $50 \mathrm{ng} / \mathrm{ml}$ was not statistically significant ( $\mathrm{p}=0.51 ;$ Fig. 2). However, there was a tendency for the survival of the patients with lower pleural effusion MMP-3 levels to be longer than that of the patients with higher pleural effusion MMP-3 levels.

\section{Discussion}

MPM shows a limited response to conventional chemotherapy and radiotherapy. Although the multi-target anti-folate pemetrexed has recently been approved as a first-line agent for use in combination with cisplatin for the treatment of MPM, survival of patients remains extremely poor (18), with a median survival duration of 8-18 months (19). Although in advanced cases, resection of the tumor only prolongs survival by approximately 3 months, it has been reported that patients with stage IA disease survive for five or more years following total resection of the tumor (20). Moreover, in several centers, potentially curative surgery combined with some form of adjuvant therapy has been performed. Early diagnosis may provide an opportunity for early treatment using new treat-

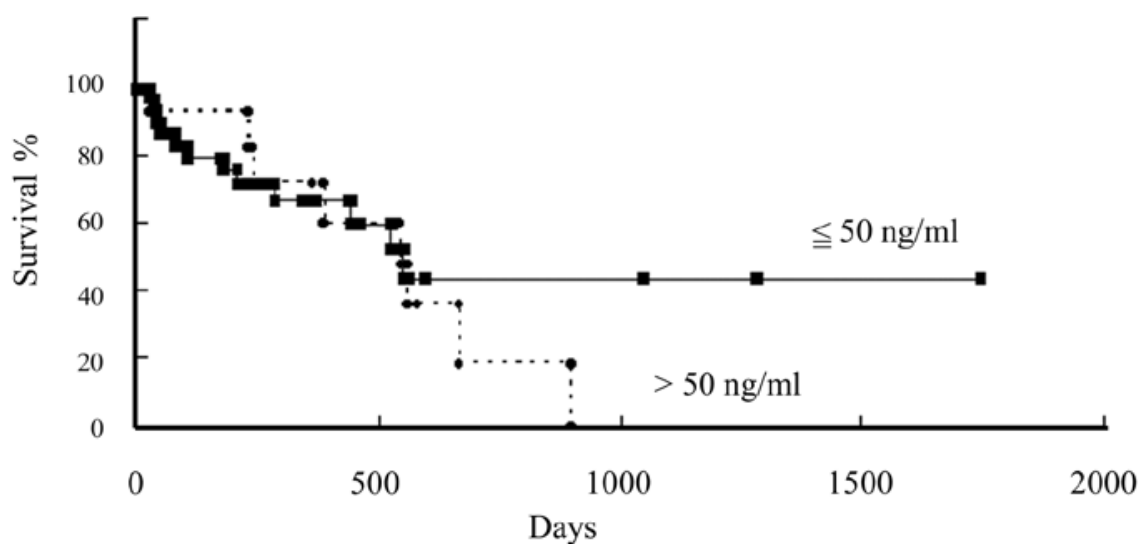

Figure 2. Survival of MPM subjects according to pleural effusion MMP-3 levels. Estimates of the probability of survival were calculated using the Kaplan-Meier method and compared using the log-rank test. MPM, malignant pleural mesothelioma; MMP-3, matrix metalloproteinase-3. 
ment regimens, although whether early intervention results in good prognosis has yet be confirmed.

Due to the difficulty of diagnosing MPM by radiological and/or histological examinations, efficient and practical pleural effusion biomarkers are required to aid the diagnosis of MPM. Several cytokines, including interleukin (IL)-6 (21), transforming growth factor (TGF)- $\beta 1$ (22-24), platelet-derived growth factor (PDGF) (25), TGF- $\alpha$ (26), and IL-8 (27), are significant in the development of MPM. Pleural effusion biomarkers for MPM, including hyaluronic acid and CYFRA 21-1, have also been reported and used to assist the diagnosis of MPM (20,28-32). The level of mesothelin-related protein (SMRP), the soluble form of mesothelin, has been reported to be a useful pleural effusion marker in MPM (33). However, little is known about their biological functions or effects on MPM cells.

A number of studies have focused on the expression of MMPs, including MMP-2 and MMP-9 in MPM cells, or in patients with MPM or lung cancer $(34,35)$. In the present study, we examined the serum and pleural effusion MMP-2 levels of MPM patients and found no significant differences between samples from patients with MPM and those from non-MPM patients (data not shown). MMP-2 and MMP-9, which are also known as gelatinase $\mathrm{A}$ and $\mathrm{B}$, respectively, cleave type IV collagen. However, MMP-3 degrades several components of the ECM, including fibronectin, laminin and collagen type IV (36). MMP-3 is also involved in tumor cell invasion and metastasis (8).

In this study, we evaluated the clinical role of the pleural effusion MMP-3 concentration as a biomarker in MPM, and demonstrated that the pleural effusion MMP-3 concentrations of patients with MPM were significantly higher than those of patients with non-malignant pleural effusion. At the optimal cut-off value of $50 \mathrm{ng} / \mathrm{ml}$, the diagnostic sensitivity of the MMP-3 pleural effusion concentration was low (30.8\%), and its negative predictive value (non-MPM patients/all patients with pleural effusion MMP-3 levels of $<50 \mathrm{ng} / \mathrm{ml}$ ) was not high $(47.1 \%)$, suggesting that the pleural effusion MMP-3 concentration cannot be used to select MPM patients from individuals with lower pleural effusion MMP-3 levels. However, its specificity was high (97.0\%) and its positive predictive value of 94.1\% (MPM patients/all patients with pleural effusion MMP-3 levels of $>50 \mathrm{ng} / \mathrm{ml}$ ), suggests that the MMP-3 concentration could be used to differentiate MPM patients from patients with higher MMP-3 pleural effusion levels. Although the difference in overall survival between the groups with lower and higher pleural effusion MMP-3 values than the cut-off point (50 ng/ml) was not statistically significant, the survival of patients with higher pleural effusion MMP-3 levels showed a tendency to be shorter than that of the patients with lower pleural effusion MMP-3 levels. This observation is compatible with the previous studies demonstrating a correlation between higher MMP-3 expression and disease progression in patients with certain malignancies (12-14). It is well known that MPM patients with higher stage and/or non-epithelioid tumor have a poor prognosis. In the present study, however, there were no significant differences in pleural effusion MMP-3 levels among disease stages as well as histological types (epithelioid versus non-epithelioid). We consider that the prognostic impact of pleural effusion MMP-3 levels needs further evaluation. From these findings, patients with high pleural effusion MMP-3 levels may be suspected of having MPM and a poor prognosis.

We evaluated the clinical role of the pleural effusion MMP-3 concentration as a biomarker in MPM, and demonstrated that patients with MPM had significantly higher pleural effusion MMP-3 levels than a population with non-malignant pleuritis involving benign asbestos pleurisy, suggesting MMP-3 to be a useful diagnostic marker of MPM. The Kaplan-Meier method revealed that the survival of the patients with higher pleural effusion MMP-3 levels showed a tendency to be shorter than that of the patients with lower pleural effusion MMP-3 levels, indicating the usefulness of MMP-3 as a prognostic marker.

\section{Acknowledgements}

We thank Ms. Hidemi Kitai for providing technical assistance. This study was supported by grants from KAKENHI, a Grant-in-Aid for Scientific Research (C) (20590936), Funds for Cancer Research from the Hyogo Prefecture Health Promotion Association and Special Coordination Funds for Promoting Science and Technology (H18-1-3-3-1).

\section{References}

1. Robinson BW, Musk AW and Lake RA: Malignant mesothelioma. Lancet 366: 397-408, 2005.

2. Robinson BW and Lake RA: Advances in malignant mesothelioma. N Engl J Med 353: 1591-1603, 2005.

3. Wagner JC, Sleggs CA and Marchand P: Diffuse pleural mesothelioma and asbestos exposure in the North; Western Cape Province. Br J Ind Med 17: 260-271, 1960.

4. Rake C, Gilham C, Hatch J, et al: Occupational, domestic and environmental mesothelioma risks in the British population: a case-control study. Br J Cancer 100: 1175-1183, 2009.

5. Selikoff IJ, Hammond EC and Seidman H: Latency of asbestos disease among insulation workers in the United States and Canada. Cancer 15: 2736-2740, 1980.

6. Liotta LA and Stetler-Stevenson WG: Metalloproteinases and cancer invasion. Semin Cancer Biol 1: 99-106, 1990.

7. Johnson LL, Dyer R and Hupe DJ: Matrix metalloproteinase. Curr Opin Chem Biol 2: 466-471, 1998.

8. Kleiner DE and Stetler-Stevenson WG: Structural biochemistry and activation of matrix metalloproteases. Curr Opin Cell Biol 5: 891-897, 1993.

9. Mukherjee S, Roth MJ, Dawsey SM, et al: Increased matrix metalloproteinase activation in esophageal squamous cell carcinoma. J Transl Med 8: 91, 2010.

10. Phromnoi K, Yodkeeree S, Anuchapreeda S, et al: Inhibition of MMP-3 activity and invasion of the MDA-MB-231 human invasive breast carcinoma cell line by bioflavonoids. Acta Pharmacol Sin 30: 1169-1176, 2009.

11. Lee EJ, Kim SY, Hyun JW, et al: Glycitein inhibits glioma cell invasion through down-regulation of MMP-3 and MMP-9 gene expression. Chem Biol Interact 185: 18-24, 2010.

12. Yeh YC, Sheu BS, Cheng HC, et al: Elevated serum matrix metalloproteinase-3 and -7 in H.pylori-related gastric cancer can be biomarkers correlating with a poor survival. Dig Dis Sci 55: 1649-1657, 2010.

13. Okamoto $\mathrm{K}$, Ishida $\mathrm{C}$, Ikebuchi $\mathrm{Y}$, et al: The genotypes of IL-1 beta and MMP-3 are associated with the prognosis of HCV-related hepatocellular carcinoma. Intern Med 49: 887-895, 2010.

14. Srivastava P, Mandhani A, Kapoor R, et al: Role of MMP-3 and MMP-9 and their haplotypes in risk of bladder cancer in North Indian cohort. Ann Surg Oncol 17: 3068-3075, 2010.

15. Harvey P, Clark IM and Jaurand MC, et al: Hepatocyte growth factor/scatter factor enhances the invasion of mesothelioma cell lines and the expression of matrix metalloproteinases. Br J Cancer 83: 1147-1153, 2000.

16. Liu Z, Ivanoff $A$ and Klominek $\mathrm{J}$ : Expression and activity of matrix metalloproteases in human malignant mesothelioma cell lines. Int J Cancer 91: 638-643, 2001. 
17. Rusch VW: A proposed new international TNM staging system for malignant pleural mesothelioma. From the International Mesothelioma Interest Group. Chest 108: 1122-1128, 1995.

18. Vogelzang NJ, Rusthoven JJ, Symanowski J, et al: Phase III study of pemetrexed in combination with cisplatin versus cisplatin alone in patients with malignant pleural mesothelioma. J Clin Oncol 21: 2636-2644, 2003.

19. Nowak AK, Lake RA, Kindler HL, et al: New approaches for mesothelioma: biologics, vaccines, gene therapy, and other novel agents. Semin Oncol 29: 82-96, 2002.

20. Pass HI, Lott D, Lonardo F, et al: Asbestos exposure, pleural mesothelioma, and serum osteopontin levels. N Engl J Med 353: 1564-1573, 2005.

21. Adachi Y, Aoki C, Yoshio-Hoshino N, et al: Interleukin-6 induces both cell growth and VEGF production in malignant mesotheliomas. Int J Cancer 119: 1303-1311, 2006.

22. Tabata C, Tabata R, Hirayama N, et al: All-trans-retinoic acid inhibits tumor growth of malignant pleural mesothelioma in mice. Eur Respir J 34: 1159-1167, 2009.

23. Fitzpatrick DR, Bielefeldt-Ohmann H, Himbeck RP, et al: Transforming growth factor-beta: antisense RNA-mediated inhibition affects anchorage-independent growth, tumorigenicity and tumor-infiltrating T-cells in malignant mesothelioma. Growth Factors 11: 29-44, 1994

24. Marzo AL, Fitzpatrick DR, Robinson BW, et al: Antisense oligonucleotides specific for transforming growth factor beta 2 inhibit the growth of malignant mesothelioma both in vitro and in vivo. Cancer Res 57: 3200-3207, 1997.

25. Versnel MA, Claesson-Welsh L, Hammacher A, et al: Human malignant mesothelioma cell lines express PDGF beta-receptors whereas cultured normal mesothelial cells express predominantly PDGF alpha-receptors. Oncogene 6: 2005-2011,1991.

26. Mórocz IA, Schmitter D, Lauber B, et al: Autocrine stimulation of a human lung mesothelioma cell line is mediated through the transforming growth factor alpha/epidermal growth factor receptor mitogenic pathway. Br J Cancer 70: 850-856, 1994.
27. Galffy G, Mohammed KA, Dowling PA, et al: Interleukin 8: an autocrine growth factor for malignant mesothelioma. Cancer Res 59: 367-371, 1999.

28. Fuhrman C, Duche JC, Chouaid C, et al: Use of tumor markers for differential diagnosis of mesothelioma and secondary pleural malignancies. Clin Biochem 33: 405-410, 2000.

29. Paganuzzi M, Onetto M, Marroni P, et al: Diagnostic value of CYFRA 21-1 tumor marker and CEA in pleural effusion due to mesothelioma. Chest 119: 1138-1142, 2001.

30. Robinson BW, Creaney J, Lake R, et al: Mesothelin-family proteins and diagnosis of mesothelioma. Lancet 362: 1612-1616, 2003.

31. Frebourg T, Lerebours G, Delpech B, et al: Serum hyaluronate in malignant pleural mesothelioma. Cancer 59: 2104-2107, 1987.

32. Schouwink H, Korse CM, Bonfrer JM, et al: Prognostic value of the serum tumour markers Cyfra 21-1 and tissue polypeptide antigen in malignant mesothelioma. Lung Cancer 25: 25-32, 1999.

33. Creaney J, Yeoman D, Naumoff LK, et al: Soluble mesothelin in effusions: a useful tool for the diagnosis of malignant mesothelioma. Thorax 62: 569-576, 2007.

34. Edwards JG, McLaren J, Jones JL, et al: Matrix metalloproteinases 2 and 9 (gelatinases $\mathrm{A}$ and $\mathrm{B}$ ) expression in malignant mesothelioma and benign pleura. Br J Cancer 88: 1553-1559, 2003.

35. Roomi MW, Monterrey JC, Kalinovsky T, et al: Modulation of MMP-2 and MMP-9 by cytokines, mitogens and inhibitors in lung cancer and malignant mesothelioma cell lines. Oncol Rep 22: $1283-1291,2009$

36. Murphy GJ, Murphy G and Reynolds JJ: The origin matrix metalloproteinases and their familial relationships. FEBS Lett 289: 4-7, 1991. 